\title{
184. Sympathetic Nerve Activities and the Paradoxical Sleep in the Decerebrate Cat
}

\author{
By Yoshiaki Iwamura, Yoshio Uchino, Seiji Ozawa, \\ and Shizuo TORII*) \\ Department of Neurophysiology, Institute of Brain Research, \\ School of Medicine, University of Tokyo \\ (Comm. by Yas Kuno, M.J.A., Sept. 12, 1966)
}

The "activated" or "paradoxical" sleep (PS), ${ }^{1,2)}$ has been a recent topic in the study of sleep. Various somatic and autonomic activities have been extensively investigated in the cat. ${ }^{3)-8)}$ The systemic blood pressure was shown to decrease during PS in intact, ${ }^{4,9), 10}$ in chronically vagotomized cats, ${ }^{10)}$ and in cats with sino-aortic deafferentation. ${ }^{9}$ A recent work of Jouvet ${ }^{3)}$ has revealed that PS occurred even in mesencephalic or pontile cats, in acute and chronic states: PS may be characterized by 1) decrease in neck muscle activity, 2) slow or spiky bursts activity (pseudo-spindle wave) of the ponto-medullary reticular formation, and 3) rapid eye movements. We used this preparation in the acute postoperative phase. The present attempt is to show the systemic blood pressure fall during PS can be observed in the decerebrate preparation, and it is accompanied by the decrease in spontaneous sympathetic nerve discharges.

Under ether anesthesia, 10 cats were decerebrated at a precollicular level using a blade and suction pump. The common carotid arteries were tied. The carotid sinus nerves, depressor and vagal nerves were cut bilaterally.

The left renal branch of the abdominal sympathetic trunk $(\mathrm{RN})^{11)}$ was dissected by retroperitoneal approach and was mounted on a pair of silver wire electrodes (interpolar distance: $2-3 \mathrm{~mm}$ ) in a warm mineral oil pool. The $\mathrm{RN}$ discharges and their integrated pattern (time const.: 4.0 sec.; cut-off frequency of the high pass filter: $8 \mathrm{cps}$ ) were recorded with an electroencephalograph (SAN'EI EG-900 type) (Fig. 1, A), and monitored by a cathode ray oscilloscope (NIHONKODEN VC-6 type). The ink-writing oscillograph (SAN'EI 1R-302 type) was also used for slower speed recording (Fig. 1, B).

Blood pressure was recorded from the left common carotid artery. Eye movements, neck muscle activity, spirogram and electrocardiogram were recorded in a conventional way. Concentric bipolar

*) Present address: Department of Physiology, Toho University, School of Medicine, Omori Nishi 5-21, Ota-ku, Tokyo. 


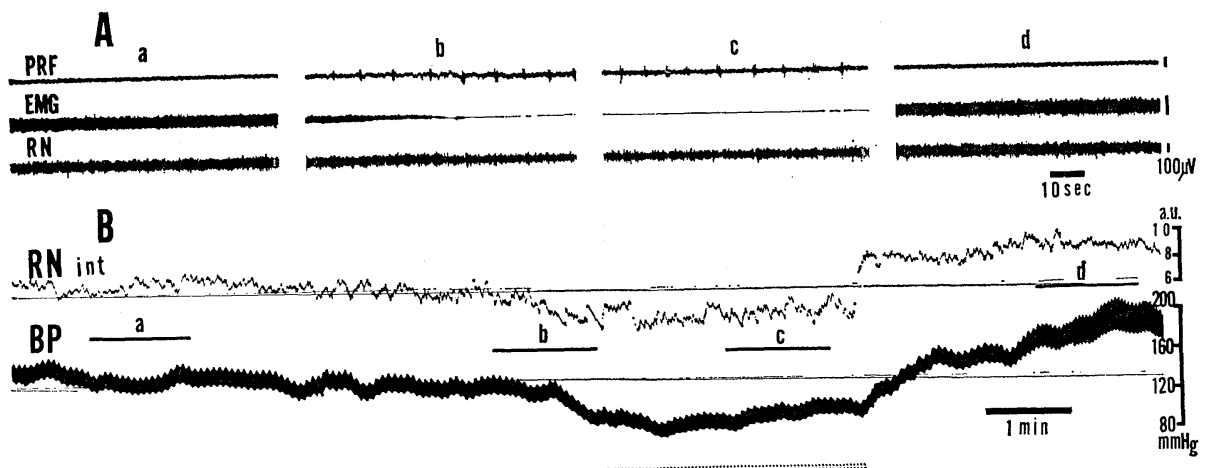

Fig. 1. A and B, polygraphic records with different paper speeds. A a, b, $c$, and $d$ were taken in respective periods indicated by black bars a-d in B. A stippled bar at the bottom of B indicates the duration of PS episode identified by the polygraph.

Abbreviations; PRF: EEG in the ponto-medullary reticular formation, EMG: neck muscle activity, RN: renal nerve discharge, RNint.: integrated record of renal nerve discharge, (a.u., arbitrary unit), BP: blood pressure.

The heart rate was $138,126,120$, and $150 /$ min and respiratory rate was $16,15,14$, and $24 / \mathrm{min}$ in the periodes $\mathrm{a}, \mathrm{b}$, c, and $\mathrm{d}$ respectively. In this case, the eye movements were not observed, on account of damaging the oculomotor nerves during operative procedures.

electrodes were inserted stereotaxically for recording in the upper and medial medullary reticular formation.

Observations were started at least 5 hours after discontinuing ether and continued for up to 20 hours. The animal was laid on its side without fixation on a wooden table. The animal remained still, being kept free from noxious stimuli. The rectal temperature was kept at $36-38^{\circ} \mathrm{c}$. A considerable amount of rigidity was observed in the extremities and in the neck muscles. The tonic neck reflex could be evoked by changing the head position. The animals showed very few spontaneous movements or postural changes which would characterize the behavioral arousal. The pupil was miotic, and nictitating membrane was relaxed. No eye movements were observed. Respiratory rate $(10-20 / \mathrm{min})$ and heart rate $(120-220 / \mathrm{min})$ were regular. The medullary electrical activity showed a low voltage fast pattern. The mean blood pressure was constant with a small fluctuation of less than $10 \mathrm{mmHg}$.

Amplitude of RN potential was less than $100-150 \mu \mathrm{V}$. There was a small and irregular fluctuation in tonic $\mathrm{RN}$ discharge, but not in phase with cardiac or respiratory cycle due presumably to sino-aortic deafferentation. ${ }^{12}$ Average amount of $\mathrm{RN}$ discharges sometimes undulated slowly with the mean level of the blood pressure with some delay in the latter (lefthand record in Fig. 1, B). A 
state with above-described signs was taken as the control, because PS always developed therefrom in the preparations observed.

The episode of PS occurred periodically with interval of $20 \mathrm{~min}$. to 1 hour in 3 cats, and it occurred only once or three times in another 2 cats. In the rest 5 cases, PS did not occur at all. We observed a total of 43 episodes of spontaneously occuring PS. The duration of each PS episode was less than 10 minutes. Fig. 1, A and B illustrate polygraphic records during a series of the control state and an episode of PS. During transition from the control state to PS the neck EMG decreased to zero, the ponto-medullary burst appeared and tonic RN discharge was reduced (Fig. 1, Ab). Change in $\mathrm{RN}$ discharge can be clearly seen in its integrated record (Fig. 1, Bb). Almost simultaneously, the blood pressure fell (Fig. 1, Bb). The decreases of both blood pressure and $\mathrm{RN}$ discharge was sustained during PS (Fig. 1, Ac, Bb-c). After PS blood pressure and RN discharge recovered up to higher levels than control (Fig. 1, Ad, Bc-d).

The heart rate decreased during PS by $0-10$ per cent, and the respiratory rate decreased by 10-15 per cent compared with control.

The lowest values of mean blood pressure were $70-129 \mathrm{mmHg}$ (average and S.D.: $119.0 \pm 15.8$ ) during the control state and 53$104 \mathrm{mmHg}(80.2 \pm 17.4)$ during PS in a total of 35 observations. The difference between the two values was significant $(\mathrm{P}<0.001)$. These values were in the similar range as the results obtained in the unrestrained chronic cats with sino-aortic deafferentation. ${ }^{9)}$

It was generally impressed that the phasic fluctuation in the blood pressure was less conspicuous in the present preparations than in chronic unrestrained cats. ${ }^{4), 10)}$ Other phasic phenomena such as rapid eye movements, twitch movements of extremities were also less remarkable, as Jouvet already noticed. ${ }^{3)}$

The concomitant decreases in the blood pressure and RN discharge suggest the decrease of the vasoconstrictor tone is one of the important factors which cause the fall in blood pressure during PS. We cannot decide at present whether the decrease in the sympathetic postganglionic outflow is attributed to the autochthonous depression in activities of the pressure center or the exaggeration of the depressor center activity. ${ }^{13)}$

\section{References}

1) W. Dement: Electroenceph. clin. Neurophysiol., 10, 291 (1958).

2) M. Jouvet, and F. Michel: J. Courjon, C. R. Soc. Biol. Paris, 153, 1024 (1959).

3) M. Jouvet: Progress in Brain Research (eds., K. Akert, C. Bally, and J. P. Schadé), Vol. 18, Elsevier Publ. Co., Amsterdam, 20-62 (1965). 
4) O. Candia, E. Favale, A. Giussani, and G. F. Rossi: Arch. ital. Biol., 100, 216 (1962).

5) O. C. Brooks, and E. Bizzi: Arch. ital. Biol., 101, 648 (1963).

6) G. Berlucchi, G. Moruzzi, G. Salvi, and P. Strata: Arch. ital. Biol., 102, 230, (1964).

7) S. Giaquinto, O. Pompeiano, and I. Somogyi: Arch. ital. Biol., 102, 245 (1964).

8) K. Kubota, Y. Iwamura, and Y. Niimi: J. Neurophysiol., 28, 125 (1965).

9) M. Guazzi, and A. Zanchetti: Science, 148, 397 (1965).

10) T. Tokizane: Progress in Brain Research (eds., T. Tokizane, and J. P. Schadé)

Vol. 21B, Elsevier publ. Co., Amsterdam, 230-268 (1966).

11) R. Engelhorn: Arch. Exp. Path. Pharmacol., 231, 219 (1957).

12) E. D. Adrian, D. W. Bronk, and G. Phillips: J. Physiol., 74, 115 (1932).

13) R. S. Alexander: J. Neurophysiol., 9, 205 (1946). 